\title{
Metrópoles, cobertura vegetal, áreas verdes e saúde
}

\author{
LUÍS FER NANDO AMATO-LOURENÇO, I, III \\ TIANA CARLA LOPES MOREIRA, I, III \\ BRUNA LARA DE ARANTES, II \\ DEMÓSTENES FERREIRA DA SILVA FILHO II \\ e THAIS MAUAD ${ }^{\text {I, III }}$
}

\section{Problematização}

$\mathrm{O}$ DESENVOLVIMENTO das grandes metrópoles é fundamentado na condensação de processos sociais e espaciais, em que a não priorização da dimensão humana deu origem a padrões urbanísticos inadequados e consolidados por um planejamento desestruturado, em contraponto às dinâmicas de um modelo urbano mais sustentável. Projeções das Nações Unidas (2004) apontam que, no ano de 2050, 66\% da população mundial habitarão áreas urbanas, gerando uma pressão sem precedentes na capacidade de suporte ambiental das grandes cidades pelo aumento no fluxo de recursos e a decorrente fragmentação na coexistência dos seus sistemas naturais e sociais. Entre as externalidades negativas associadas aos processos de apropriação e expansão acelerada das grandes metrópoles, incluem-se a supressão da sua cobertura vegetal e a decorrente diminuição de áreas verdes disponíveis à fruição pública.

A infraestrutura verde pode ser definida como uma rede de espaços verdes interconectados que conservam valores naturais de um ecossistema e que provêm benefícios às populações humanas (Coutts; Hahn, 2015). Pode ser composta por parques, florestas, praças, hortas comunitárias e outras formas de paisagens naturais públicas ou privadas. Nas cidades, incluem-se também a arborização urbana e os tetos verdes.

Cada vez mais são reconhecidos os efeitos benéficos que o contato com a natureza gera à saúde humana. Os efeitos positivos do contato com áreas verdes foram observados em relação a longevidade, doenças cardiovasculares, obesidade, saúde mental, qualidade do sono, recuperação de doenças e desfechos de natalidade. A maioria desses estudos é europeia ou norte-americana, não existindo ainda dados nacionais ou da cidade de São Paulo. Nesses países, os efeitos mais apreciados são observados em áreas urbanas e em classes socioeconômicas mais baixas.

Os mecanismos pelos quais as áreas verdes são benéficas à saúde não estão bem esclarecidos, e provavelmente devem ser multifatoriais. Diminuição do estres- 
se/aumento do relaxamento, contatos sociais e atividade física têm sido propostos como prováveis mecanismos. Fatores ambientais como diminuição da temperatura e ruídos, aumento da umidade e captura de material particulado certamente têm uma influência nos efeitos fisiológicos protetores dos ambientes verdes.

Nesta revisão, apresentamos uma série de estudos enfocando a associação entre áreas verdes e saúde.

\section{Serviços ecossistêmicos das áreas verdes e coberturas vegetais ${ }^{1}$}

As áreas verdes possuem diversos benefícios, tanto ecossistêmicos como salutogênicos. Entre os benefícios ecossistêmicos podemos citar a diminuição de temperaturas (Oliveira et al., 2011) resultando em um decréscimo de ilhas de calor (Solecki et al., 2005) além do consumo de eletricidade (McPherson; Simpson, 2003). Além disso, são gerados benefícios como a diminuição de escoamento superficial da água (runoff), concentração de poluentes atmosféricos, redução de ruído, impacto de ventos, incidência solar em pavimentos e construções (O'Dell et al., 1977; Nowak et al., 2007; Fang; Ling, 2005; McPherson; Muchnick, 2005) e como estoque de carbono (Davies et al., 2011). A vegetação possui ainda funções estéticas e recreativas. Propriedades próximas de áreas verdes ou possuindo vegetação tendem a possuir maior valor no mercado imobiliário. De acordo com o serviço florestal americano os benefícios das áreas verdes urbanas ou florestas urbanas proporcionam uma economia três vezes maior que o custo da manutenção destas áreas (USDA Forest Service, 2016).

\section{Regulação térmica}

A vegetação diminui temperaturas devido à sua evapotranspiração e produção de sombras. O aumento de $10 \%$ na cobertura vegetal em Munique durante o verão é capaz de diminuir até $1,4^{\circ} \mathrm{C}$ a temperatura superficial (Pauleit; Duhme, 2000). O arrefecimento da temperatura também pode resultar na economia de U\$ 18,5 milhões/ano devido à redução da utilização de energia elétrica, conforme descrito por Simpson (1998) para a cidade de Sacramento (Califórnia - Estados Unidos).

As árvores possuem efeito protetor de pavimentos e construções produzindo sombras e diminuindo a incidência direta de sol na pavimentação viária. O excesso de calor é responsável pela degradação acelerada de matérias de revestimento do solo, como rachaduras, sulcos e buracos. McPherson e Muchnick (2005) verificaram que nas vias com presença de sombra na cidade de Modesto (Califórnia) existia uma maior conservação do asfalto viário, resultando em uma economia para os cofres públicos de U\$ $7,13 / \mathrm{m}^{2}$.

\section{Escoamento superficial}

A vegetação diminui o impacto da água no solo assim como o seu escoamento superficial. Zhang et al. (2015) observaram que a redução de $199 \mathrm{~km}^{2}$ de áreas verdes na cidade de Pequim, entre os anos 2000 e 2010, acarretou no aumento do escoamento superficial da água de $17 \%$ para $23 \%$. A redução do 
runoff pode diminuir as enchentes e ainda melhorar a qualidade das águas pluviais que são direcionadas para os rios. Além disso, a cobertura vegetal também possui a capacidade de filtrar poluentes associados ao runoff como demonstrado por Coutts e Hahn (2015).

\section{Modulação de doenças infecciosas transmitidas por vetores}

Reduções de áreas verdes têm sido associadas à alteração de características do meio físico, como a desregulação do microclima local. Essa modificação pode afetar diretamente a composição da fauna de vetores responsáveis por doenças infecciosas. A essas mudanças são atribuídos aumento na taxa do desenvolvimento larval, frequência, deriva genética de populações e taxas de sobrevivência de mosquitos pertencentes à família dos Culicideos (responsáveis pela transmissão de doenças como dengue, febre amarela, malária, filarioses e encefalites). Afrane et al. (2005) relataram que o aumento na temperatura de $0,5{ }^{\circ} \mathrm{C}$ foi associado com o aceleramento do ciclo reprodutivo da espécie Anopheles, mosquito transmissor de doenças como a malária e a filariose.

\section{Qualidade do ar}

As áreas verdes possuem funções de filtro de poluentes, tanto para o material particulado quanto para gases. Nowak et al. (2006) utilizaram modelagem computacional para avaliar o quanto florestas urbanas de 55 cidades dos Estados Unidos removeram de poluentes da atmosfera. A absorção de poluentes variou entre as cidades e foi retirado da atmosfera um total de $711.000 \mathrm{t} / \mathrm{m}^{3}$ de $\mathrm{O}_{3}$, $\mathrm{PM}_{10}, \mathrm{NO}_{2}, \mathrm{SO}_{2}, \mathrm{CO}$. No ano 2010, Nowak et al. (2014) observaram que a área coberta por vegetação nas cidades americanas foi responsável pela remoção de 17,4 milhões de toneladas gerando uma economia de U\$ 6,8 bilhões com a redução da utilização de sistemas de saúde e dias de trabalho perdidos.

A contribuição da vegetação na atenuação da poluição atmosférica também pode ocorrer de forma indireta como na geração de $\mathrm{O}_{3}$ devido à redução da temperatura. Durante a transpiração da vegetação ocorre a diminuição da temperatura e o aumento da umidade relativa do ar, diminuindo a emissão de hidrocarbonetos (McPherson et al., 1998).

\section{Redução de ruídos}

Áreas verdes urbanas também têm sido associadas à atenuação de ruídos de diferentes frequências, agindo como barreiras verticais (Yang et al., 2010). Gidlöf-Gunnarsson e Öhrström (2007) avaliaram moradores residindo em locais de alta e baixa intensidade de ruídos atribuídos ao tráfego veicular. Os resultados mostraram que locais com maiores áreas de vegetação tendem a reduzir incômodos em longo prazo gerados pelos ruídos veiculares (independentemente da intensidade) e a prevalência de sintomas relacionados ao estresse psicossocial.

\section{Valorização imobiliária}

Muitos dos benefícios atribuídos à cobertura vegetal são difíceis de ser valorados (exemplo, embelezamento, privacidade e bem-estar), porém alguns des- 
ses benefícios podem ser relacionados ao valor de mercado da propriedade. $\mathrm{Na}$ cidade de Portland (Oregon) a presença de 0,55 árvore na frente da residência e a existência de $84 \mathrm{~m}^{2}$ de cobertura vegetal a menos de $100 \mathrm{~m}$ da propriedade aumentam o valor imobiliário em 3\% (Donovan; Butry, 2010).

Escobedo et al. (2015) analisaram a relação entre o valor da propriedade e a presença de áreas verdes em quatro cidades no estado da Flórida, nos Estados Unidos. Foi observado que a adição de uma árvore no lote aumenta o valor da propriedade em US $\$ 1.586,00$, porém a troca da área gramada de $25 \%$ para $75 \%$ diminuiu o valor da propriedade em US\$271,00.

\section{Produção de alimentos}

Árvores e áreas verdes plantadas, como as hortas urbanas, fornecem frutas e pólen para animais e insetos, que por sua vez são responsáveis pela polinização e pelo equilíbrio biológico local, além de constituírem locais de refúgios para a microfauna e a avifauna. $\mathrm{O}$ valor econômico anual do serviço de polinização prestado pelas abelhas, somente nos Estados Unidos, é estimado entre US\$ 14,6 e US\$ 40 bilhões (Morse; Calderone, 2000).

A popularização e ampliação do número de hortas urbanas comunitárias nos últimos anos vem contribuindo com o aumento de áreas verdes públicas e os decorrentes benefícios ambientais, urbanísticos, sociais e de saúde pública à população. Adjunto à produção de frutas, hortaliças e vegetais cultivados localmente é oferecida uma série de serviços ecossistêmicos considerados essenciais nos processos de recriação da noção de unidade cidade-campo, reinventando a multifuncionalidade da paisagem e permitindo que conceitos e atitudes sobre sustentabilidade sejam compartilhados e integrados à sociedade. Nesse sentido, a criação de novas hortas comunitárias é justificada pela forma nobre de se utilizar espaços públicos pouco aproveitados, permitindo que locais como áreas intersticiais não construídas e superfícies urbanas/periurbanas sejam valorizadas.

Os efeitos na saúde humana são diversos e em sua maioria estão relacionados com algum dos benefícios ecossistêmicos, exemplo: diminuição da concentração de poluentes atmosféricos pelas plantas e melhora de doenças respiratórias. A Figura 1 ilustra as principais vias nas quais as áreas verdes podem contribuir positivamente para a saúde humana. 


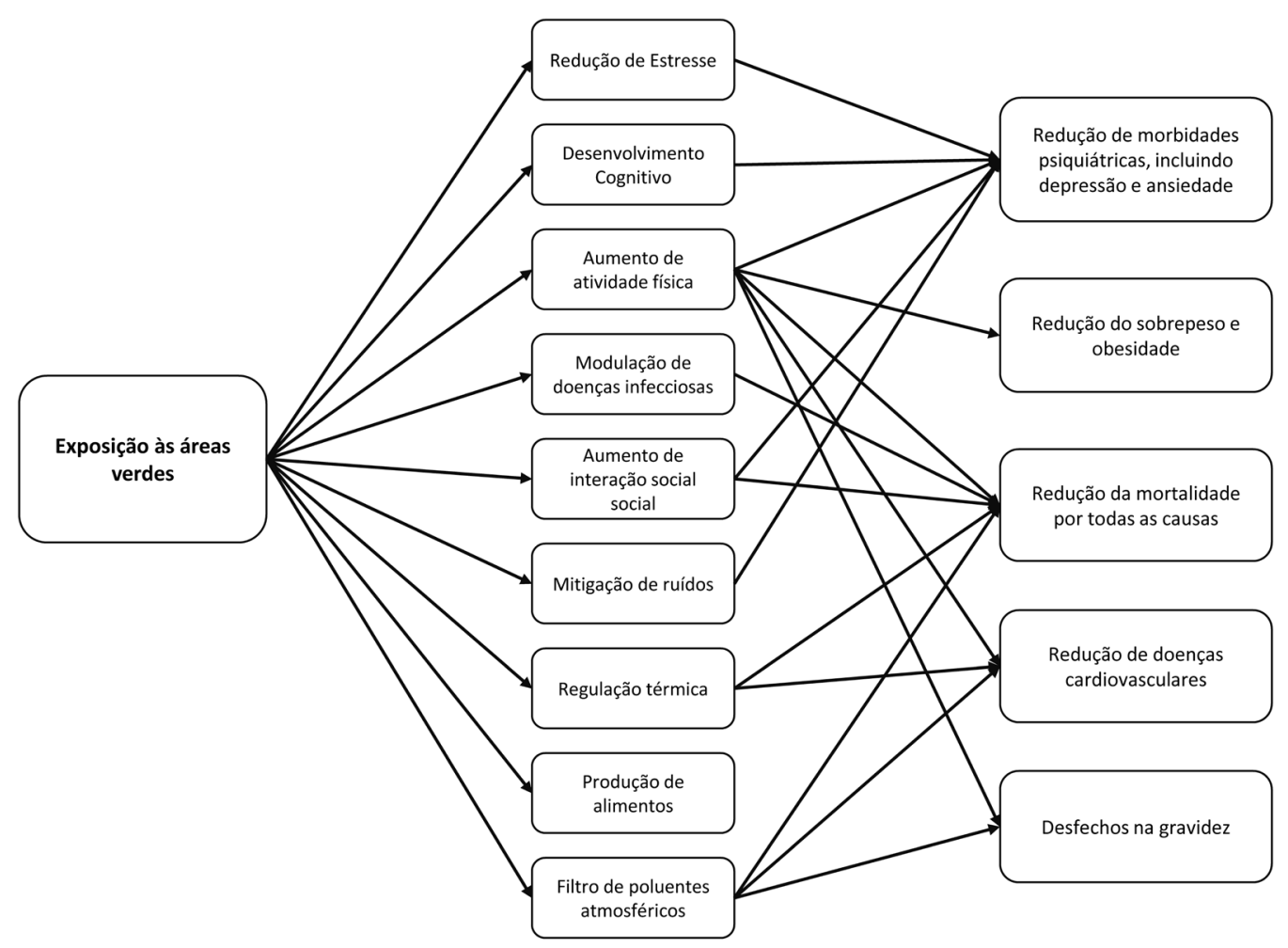

Figura 1 - Vias em que exposições a áreas verdes podem resultar em desfechos na saúde (Adaptado de James et al., 2015).

\section{Áreas verdes e efeitos associados à saúde humana}

Inúmeros estudos epidemiológicos e experimentais têm demonstrado uma possível associação entre a existência de áreas verdes intra/extraurbanas e uma série de efeitos benéficos à saúde mental e física da população.

\section{Saúde mental}

A ausência de áreas verdes em locais residenciais tem sido associada a um dos fatores contextuais que possuem papel etiológico na ocorrência de transtornos mentais comuns ${ }^{2}$ (Araya et al., 2007). Nesse contexto, o estudo transversal conduzido por Van der Berg et al. (2016) em quatro cidades europeias com 3.748 participantes demonstrou que quanto maior o tempo gasto visitando áreas verdes, maiores foram os escores de avaliação para saúde mental, independentemente dos contextos culturais e climáticos. Astell-Burt et al. (2014) verificaram em um estudo longitudinal, com 65.407 adultos ( $>15$ anos), que o aumento de espaços verdes próximos ao local de residência foi associado a menores riscos de morbidades psiquiátricas entre homens $>35$ anos e mulheres $>41$ anos. Thompson et al. (2012) associaram níveis de exposição de adultos às áreas verdes (ajustados por variáveis socioeconômicas e demográficas) ao declínio de cortisol salivar diurno como biomarcador da variação nos níveis de estresse. 


\section{Desenvolvimento cognitivo e atenção}

O estudo realizado por Dadvand et al. (2015) investigou a relação existente entre a exposição a espaços verdes e o desenvolvimento cognitivo com 2.593 crianças frequentando escolas primárias. Os achados mostraram o progresso na memória de trabalho e a diminuição na desatenção durante o aprendizado associados à presença de áreas verdes no local de ensino e em seu entorno. Os benefícios à saúde citados podem ser explicados, como pela capacidade que as áreas verdes possuem em reduzir ruídos ambientais que impactariam negativamente no desenvolvimento cognitivo. Além disso, a presença de espaços verdes tem sido associada ao aumento de atividades físicas, que por sua vez está diretamente relacionado com melhor função cognitiva em crianças.

\section{Obesidade e sedentarismo}

Dadvand et al. (2014) conduziram um estudo com 3.178 crianças escolares relatando que o aumento de áreas verdes no entorno das suas residências foi associado com menor prevalência relativa (11\%-19\%) no sobrepeso/obesidade e comportamento sedentário. Porém, os resultados apontaram que viver perto de parques foi associado com o aumento de $60 \%$ na prevalência relativa de asma atual, fato esse atribuído à vegetação anemófila local (polinizadas pelo vento) conhecidas por suas propriedades alergênicas. Portanto, a escolha de espécies adequadas possui um papel crítico no planejamento de espaços verdes para que sejam obtidos os benefícios associados.

\section{Envelhecimento}

Kweon et al. (1998) relataram que a exposição às áreas verdes em regiões intraurbanas de Chicago está associada a melhor integração social e fortalecimento de laços sociais entre idosos. Nesse sentido, Takano et al. (2002) demonstraram em um estudo de coorte a associação de espaços verdes abertos a caminhantes nas proximidades de residências e a longevidade de idosos na região metropolitana de Tóquio. Os resultados evidenciaram um efeito protetor na longevidade de idosos urbanos, independentemente de sua idade, sexo, estado civil e nível socioeconômico, associados a viver em áreas verdes de fácil acesso.

\section{Morbimortalidade}

Wilker et al. (2014) relataram que a proximidade residencial de áreas verdes foi associada a maiores taxas de sobrevivência após acidente vascular cerebral isquêmico em modelos multivariados ajustados para indicadores de histórico médico, fatores demográficos e socioeconômicos. Villeneuve et al. (2012) realizaram um estudo de coorte com 575 mil adultos > 35 anos, residindo em dez áreas urbanas de Ontário, Canadá. Os resultados demonstraram que o aumento interquartil de áreas verdes em um buffer de $500 \mathrm{~m}$ do local de residência foi associado a redução de mortalidade não acidental. Para causas específicas de mortalidade foi verificado um efeito protetor para desfechos respiratórios (risco relativo: 0,91). Maas et al. (2006), utilizando entrevistas com 250.782 participantes holandeses, evidenciaram que a porcentagem de espaço verde (espaço 
verde urbano, espaço rural, espaço verde natural) dentro de um raio de $1 \mathrm{~km}-3$ $\mathrm{km}$ da residência foi associada a menores taxas de autodeclaração de adoecimentos. Tamosiunas et al. (2014) realizaram um estudo tipo coorte com 5.112 participantes com idade entre 45 e 72 anos e obtiveram que a prevalência de fatores de risco cardiovascular e de diabetes mellitus foi significativamente menor entre os usuários do parque do que entre os não usuários.

Um estudo sobre prevalências de asma com crianças de 4-5 anos e crianças abaixo de 15 anos observou que o aumento da densidade de árvores para $343 \mathrm{~km}^{2}$ foi associado a uma diminuição da prevalência de asma em 24\% (risco relativo: 0,76 ). Depois de ajustar para os fatores de confusão foi estimado que o aumento para a mesma densidade de árvores foi responsável pela diminuição de 29\% da prevalência (risco relativo: 0,71) (Lovasi et al., 2008).

\section{Desfechos nos nascimentos e desenvolvimento fetal}

Os achados de Agay-Shay et al. (2014), em um estudo de coorte realizado com 39.132 nascidos vivos, demonstraram associações negativas entre a proximidade de espaços verdes durante a gravidez e baixo peso ao nascer (razão de chances: 0,85). Grazuleviciene et al. (2014) realizaram um estudo com 3.416 mulheres no primeiro trimestre de gravidez, residentes na cidade de Kaunas, na Lituânia, investigando a influência da distância entre a residência e parques públicos com valores de pressão arterial. Foi obtida uma associação positiva entre o aumento da distância às áreas verdes (considerando buffers de 300,>300-1.000, e $>1.000 \mathrm{~m}$ ) com o aumento da pressão arterial, ajustando para idade, escolaridade, condição socioeconômica, tabagismo passivo, IMC, doença crônica e estresse (razão de chances: 1,74 para o mais distante e 1,37 para a distância mais próxima). O estudo de Dadvand et al. (2012) demonstrou que o aumento interquartil de áreas verdes considerando buffers de $500 \mathrm{~m}$ foi associado com o aumento no peso de nascidos vivos (44,2 g) e aumento na circunferência craniana (1,7 mm).

\section{A cidade de São Paulo: cobertura vegetal, áreas verdes e vulnerabilidade social}

A cobertura vegetal na cidade de São Paulo possui distribuição desigual e corresponde a cerca de $42,2 \%$ do seu território de $1.521,110 \mathrm{~km}^{2}$ (Prefeitura de São Paulo, 2014). A subprefeitura de Parelheiros concentra 49,64\% do total de cobertura vegetal no município, em contraponto aos $11 \%$ totalizados nas 12 subprefeituras da zona leste da cidade.

Dados da Secretaria do Verde e Meio Ambiente do município de São Paulo (2014) demonstram que as subprefeituras com menores índices de cobertura vegetal no município, no ano de 2013 , foram: Itaim Paulista $\left(2,24 \mathrm{~m}^{2} / \mathrm{hab}\right)$, São Miguel $\left(3,43 \mathrm{~m}^{2} / \mathrm{hab}\right)$ e Vila Maria/Vila Guilherme $\left(3,91 \mathrm{~m}^{2} / \mathrm{hab}\right)$, enquanto Parelheiros $\left(2.289,73 \mathrm{~m}^{2} / \mathrm{hab}\right)$, Perus $\left(247,24 \mathrm{~m}^{2} / \mathrm{hab}\right)$, Tremembé/ Jaçanã $\left(151,31 \mathrm{~m}^{2} / \mathrm{hab}\right)$ registraram os maiores valores na cidade. Em contraponto, nas subprefeituras com os maiores valores de cobertura vegetal estão localizados parques estaduais, como o da Serra do Mar e da Cantareira, não re- 
fletindo necessariamente áreas de real uso direto e fruição pública. Quando não são contabilizadas as áreas de parques estaduais, as subprefeituras de Parelheiros e Tremembé/Jaçanã possuem respectivamente os menores índices de áreas de lazer em parques e praças por habitante $\left(0,28\right.$ e $\left.0,32 \mathrm{~m}^{2} / \mathrm{hab}\right)$.

A análise temporal dos índices de áreas verdes públicas por habitante (Figura 2) demonstra pouca variação percentual entre os anos 2009-2013 (10,3\%) não sendo estatisticamente diferentes entre os anos analisados. ${ }^{3}$ Nesse sentido, apenas cinco subprefeituras possuem o valor recomendado pela Sociedade Brasileira de Arborização Urbana (SBAU, 1996) de $15 \mathrm{~m}^{2} /$ habitante, considerado o índice mínimo associado à qualidade de vida da população (Figura 3). Esse cenário sinaliza a urgência na criação e ampliação de áreas verdes ajustadas ao crescimento demográfico e ao plano diretor municipal.

A distribuição espacial desigual de cobertura vegetal no município quando comparadas ao Índice Paulista de Vulnerabilidade Social ${ }^{4}$ (Figura 4) demonstra grandes disparidades. As subprefeituras com maiores índices de cobertura vegetal, como Parelheiros, Jaçanã/Tremembé e Perus, apresentam classificações de alta vulnerabilidade. Em contraste, no extremo leste da cidade são observados baixos valores de cobertura vegetal e altos índices. A ausência de estudos associativos que busquem quantificar a magnitude da presença de áreas verdes na qualidade de vida da população destas áreas é preponderante para responder perguntas como: Qual o impacto das áreas verdes na saúde da população considerando os diferentes índices de vulnerabilidade e distribuição de cobertura vegetal?

Além disso, um estudo holandês de 2012 (Van Dillen et al., 2012) mostra que não só a quantidade, mas a qualidade das áreas verdes têm um papel aditivo importante nos desfechos relacionados à saúde. Também de modo importante, não só a presença de grandes áreas verdes, mas também a arborização urbana se revelaram fatores consideráveis nos desfechos positivos de saúde.

Para a cobertura arbórea da cidade foram extraídos dados do mapeamento temático a partir de imagens do satélite Quickbird do ano 2009 e comparados com dados obtidos a partir de classificação temática de imagens do satélite Ikonos de 2002. Foi observada uma perda de cobertura arbórea desigual para diferentes regiões da cidade (Abreu, 2013). As regiões com maiores perdas foram as das microbacias do córrego Uberaba (7,96\%), Mooca (4,06\%) e Tiburrinho $(2,06 \%)$, enquanto outras microbacias na mesma região central obtiveram ampliação da cobertura arbórea como Dreno do Brooklin (15,01\%), Pacaembu $(9,27 \%)$ e Fortunato Ferraz $(9,45 \%)$. Além disso, foi identificada a substituição da classe solo exposto por construções e aumento do sombreamento na região, o que condiz com a possibilidade de verticalização dessas áreas. Segundo Dobbert (2015) um aumento de $10 \%$ na área em cobertura arbórea possibilita uma redução de aproximadamente $1{ }^{\circ} \mathrm{C}$ beneficiando o conforto e a saúde dos habitantes do local. 


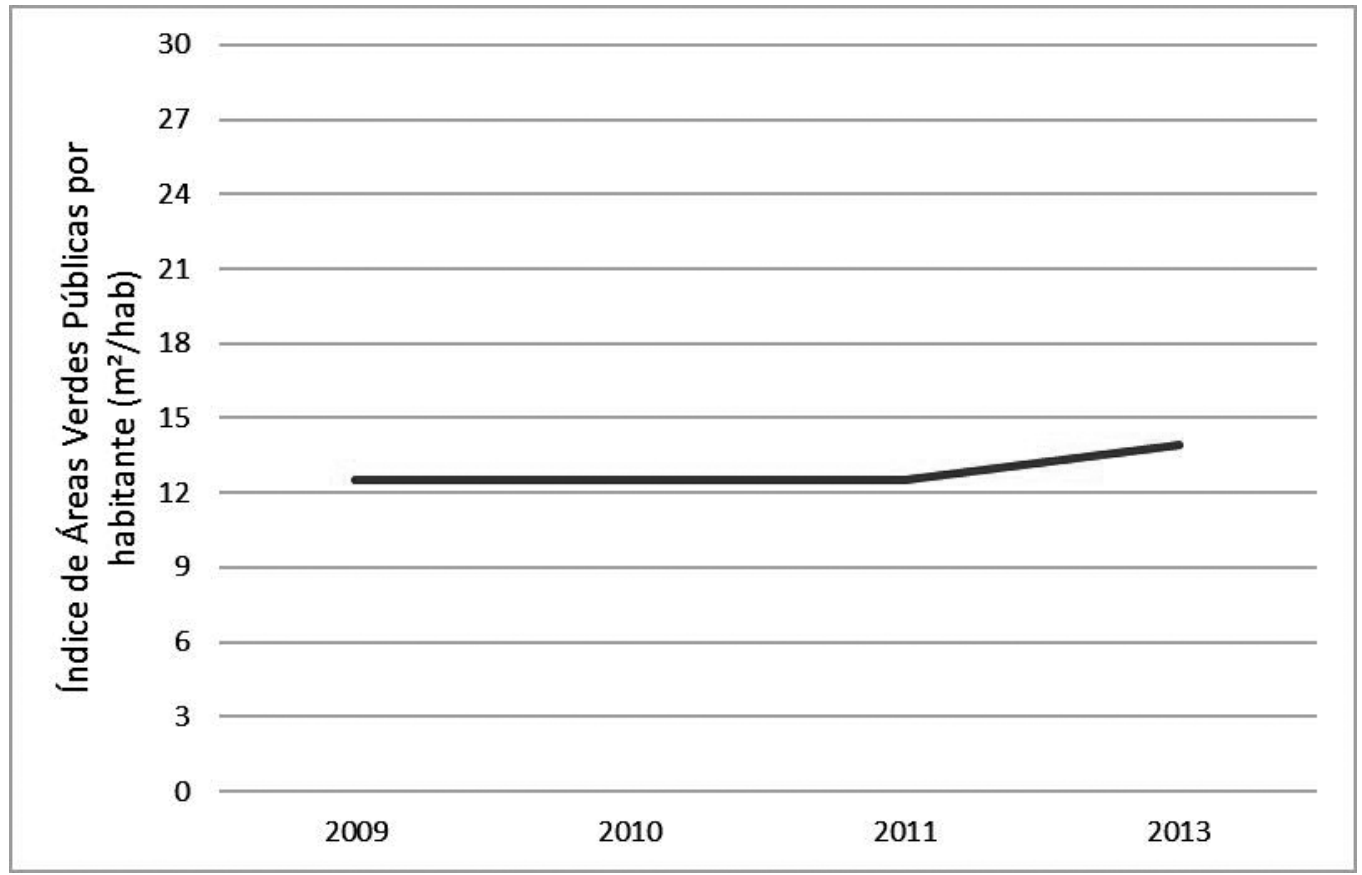

Fonte dos dados: Prefeitura de São Paulo (2014).

Figura 2 - Índice de áreas verdes públicas por habitante na cidade de São Paulo. ${ }^{5}$

\section{Índice de Áreas Verdes por habitante ( $\left.\mathrm{m}^{2} / \mathrm{hab}\right)$}

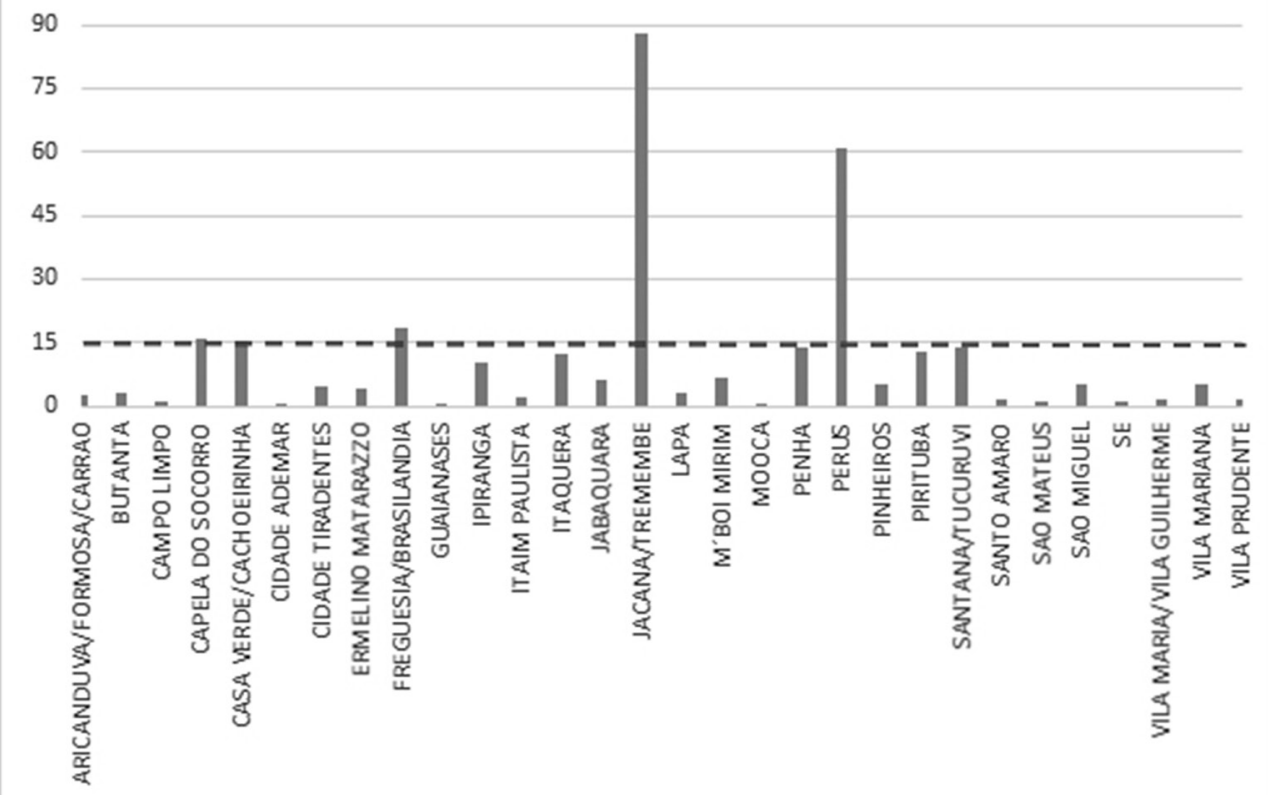

Fonte dos dados: Prefeitura de São Paulo (2014).

Figura 3 - Índice de áreas verdes por habitante e subprefeitura da cidade de São Paulo. Não foi incluída a subprefeitura de Parelheiros (IAVP $=312,82 \mathrm{~m}^{2} / \mathrm{hab}$ ) para melhor visualização do gráfico. 


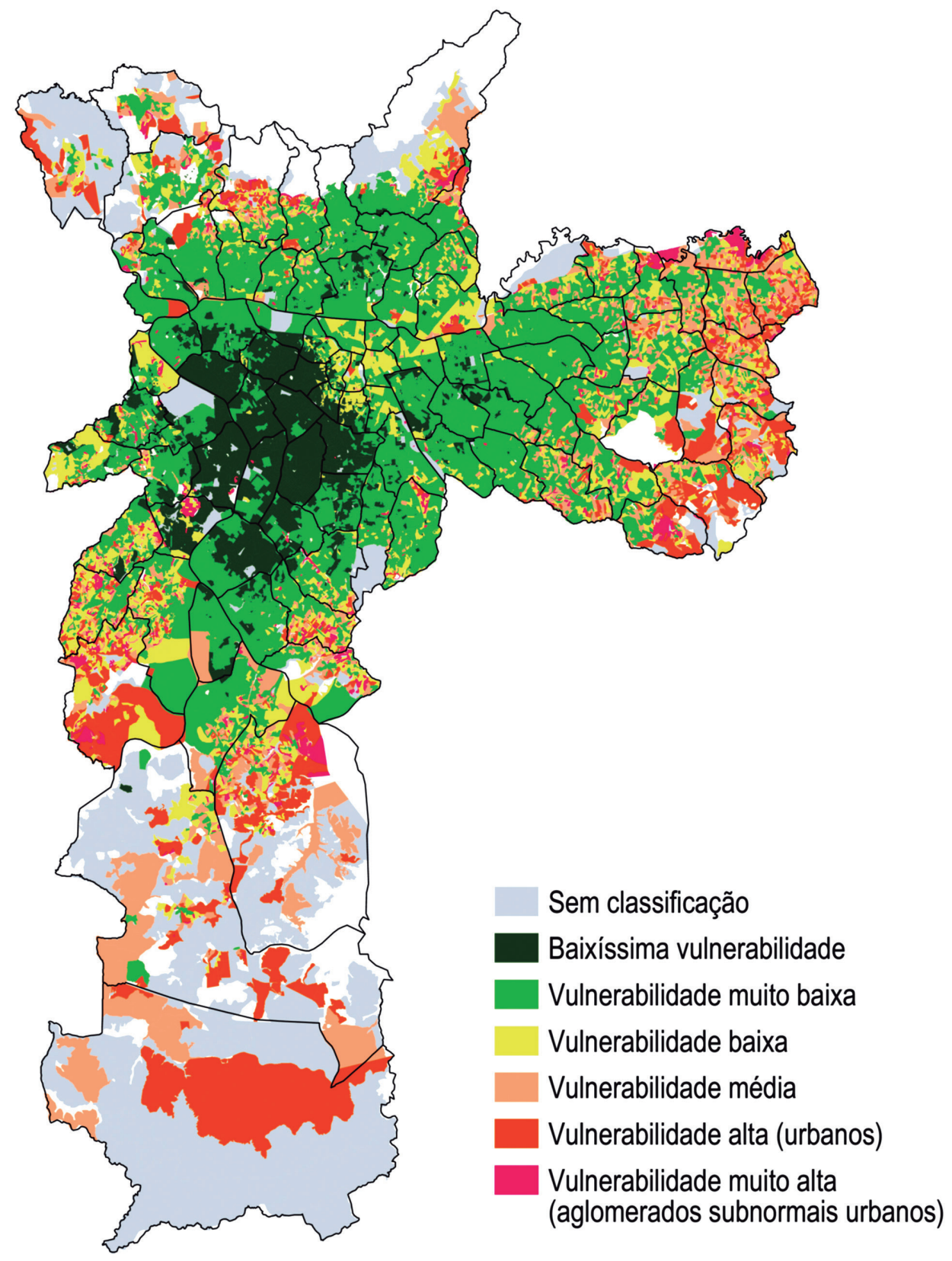

Fonte: Fundação Seade (2013).

Figura 4 - Índice Paulista de Vulnerabilidade e índice de áreas verdes ( $\mathrm{m}^{2} /$ habitante).

Na Figura 5 pode-se observar a desigualdade na cobertura arbórea com destaque para regiões pouco urbanizadas e florestais no norte e no sul do município e pouca cobertura na zona leste. A cobertura arbórea inserida no tecido urbano é mais eficiente nos bairros jardins e muito deficiente na periferia e zona leste da cidade (Silva Filho, s. d.). 


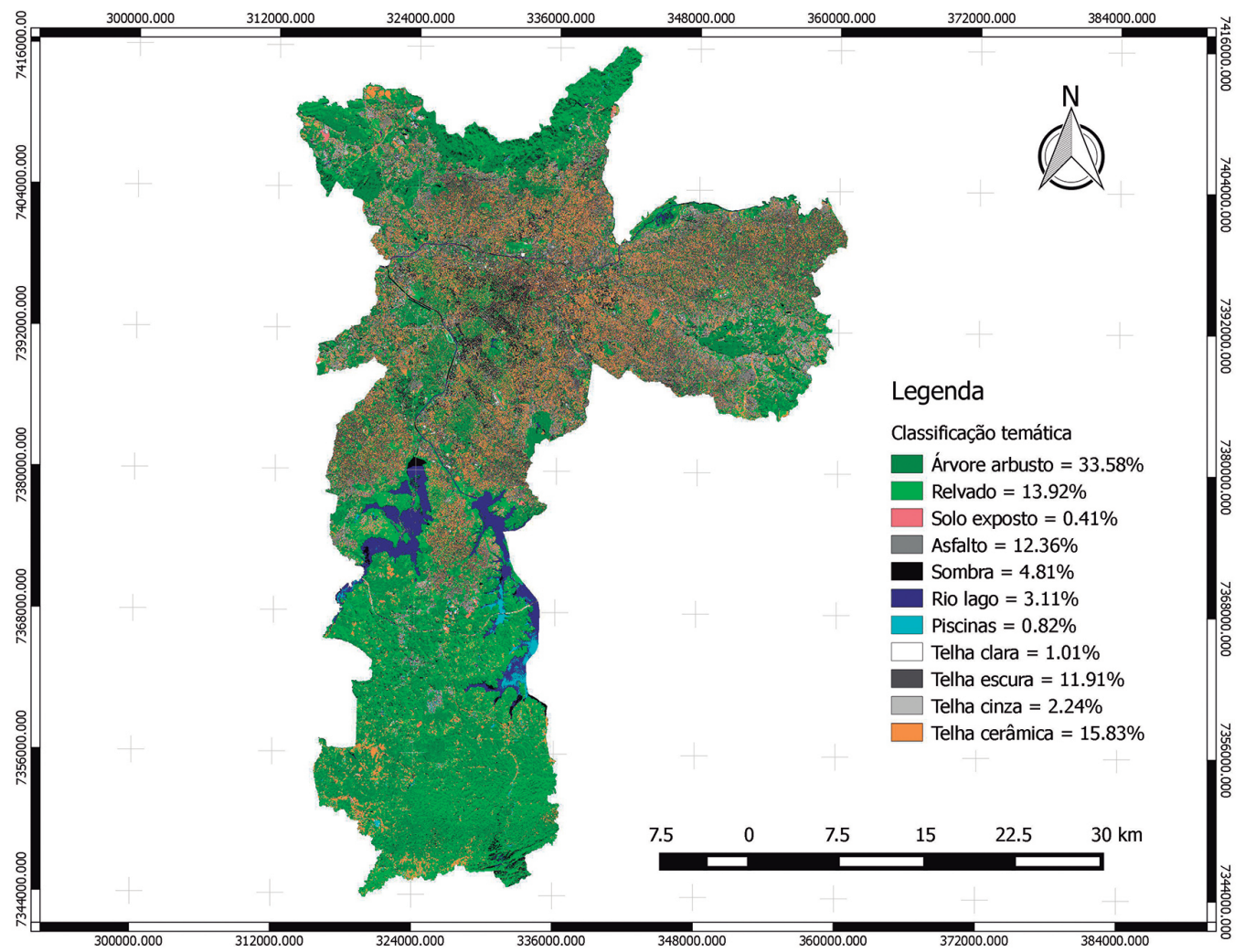

Fonte: Silva Filho (s. d.).

Figura 5 - Mapeamento temático da cobertura arbórea da cidade de São Paulo em 2010.

Comparando os dados do mapa de vulnerabilidade com o mapa de cobertura arbórea pode-se ver que a área de baixíssima vulnerabilidade corresponde às áreas com maior cobertura arbórea dispersa no tecido urbano da cidade, presentes nos bairros jardins. As regiões periféricas de alta vulnerabilidade possuem ocupação caracterizada pela autoconstrução em núcleos urbanos com intensa ocupação, pouca infraestrutura urbana e distantes dos empregos que estão localizados em áreas bem estruturadas e com baixa vulnerabilidade como descrito por Rolnik et al. (2015).

Com base nos mapeamentos de Silva Filho (s. d.) obteve-se a Tabela 1 com as coberturas arbóreas por habitante usando como dados demográficos os fornecidos pela Prefeitura de São Paulo (2016).

Os dados são semelhantes aos da cobertura vegetal, porém referentes somente à cobertura arbórea. Parelheiros possui as melhores proporções evidenciando a presença de vazios urbanos florestais e não a disponibilidade de boa arborização em vias públicas e praças. Já Butantã e Pinheiros obtiveram boas relações, embora com coberturas por habitante ainda baixas. 
Tabela 1 - Porcentagem de copas de árvores nas subprefeituras da cidade de São Paulo e de metro quadrado de copas de árvores por habitante (Copa árvore $\left(\mathrm{m}^{2}\right)$ /população) obtidos a partir de dados da Prefeitura de São Paulo (2016) e mapeamento temático de (Silva Filho, s. d.)

\begin{tabular}{|c|c|c|c|c|}
\hline Subprefeituras & $\begin{array}{c}\text { Copa de árvore } \\
(\%)\end{array}$ & $\begin{array}{c}\text { Copa de árvore } \\
\left(\mathrm{m}^{2}\right)\end{array}$ & $\begin{array}{l}\text { População } \\
\text { (2010) }\end{array}$ & $\begin{array}{c}\text { Copa árvore }\left(\mathrm{m}^{2}\right) \\
\text { /População }\end{array}$ \\
\hline Aricanduva & $12,42 \%$ & 26712 & 267.702 & 0,10 \\
\hline Butantã & $34,10 \%$ & 191301 & 428.217 & 0,45 \\
\hline Campo Limpo & $27,81 \%$ & 102051 & 607.105 & 0,17 \\
\hline Capela do Socorro & $28,41 \%$ & 381302,3 & 594.93 & 0,64 \\
\hline Casa Verde & $26,17 \%$ & 69878 & 309.376 & 0,23 \\
\hline Cidade Ademar & $22,36 \%$ & 68654 & 410.998 & 0,17 \\
\hline Cidade Tiradentes & $26,42 \%$ & 39637 & 211.501 & 0,19 \\
\hline Ermelino Matarazo & $20,18 \%$ & 30475 & 207.509 & 0,15 \\
\hline Freguesia do Ó & $32,60 \%$ & 102694 & 407.245 & 0,25 \\
\hline Guaianases & $23,10 \%$ & 41118 & 268.508 & 0,15 \\
\hline Ipiranga & $23,78 \%$ & 89162 & 463.804 & 0,19 \\
\hline Itaim Paulista & $18,00 \%$ & 39064 & 373.127 & 0,10 \\
\hline Itaquera & $34,34 \%$ & 186470 & 523.848 & 0,36 \\
\hline Jabaquara & $17,32 \%$ & 24424 & 223.78 & 0,11 \\
\hline Jaçanã & $46,11 \%$ & 295544 & 291.867 & 1,01 \\
\hline Lapa & $19,83 \%$ & 79520 & 305.526 & 0,26 \\
\hline M'boi Mirim & $23,11 \%$ & 143522 & 563.305 & 0,25 \\
\hline Mooca & $11,73 \%$ & 41282 & 343.98 & 0,12 \\
\hline Parelheiros & $53,47 \%$ & 1890069 & 139.441 & 13,55 \\
\hline Penha & $20,43 \%$ & 87423 & 474.659 & 0,18 \\
\hline Perus & $48,20 \%$ & 275700 & 146.046 & 1,89 \\
\hline Pinheiros & $28,04 \%$ & 88899 & 289.743 & 0,31 \\
\hline Pirituba & $38,07 \%$ & 208251 & 437.592 & 0,48 \\
\hline Santana & $30,85 \%$ & 107064 & 324.815 & 0,33 \\
\hline Santo Amaro & $27,85 \%$ & 104428 & 238.025 & 0,44 \\
\hline São Matheus & $27,89 \%$ & 127714 & 426.764 & 0,30 \\
\hline São Miguel & $18,09 \%$ & 43952 & 369.496 & 0,12 \\
\hline Sé & $16,16 \%$ & 42344 & 431.106 & 0,10 \\
\hline Vila Maria & $11,04 \%$ & 29158 & 297.713 & 0,10 \\
\hline Vila Mariana & $22,91 \%$ & 60708 & 344.632 & 0,18 \\
\hline Vila Prudente & $13,08 \%$ & 43564 & 531.113 & 0,08 \\
\hline
\end{tabular}


Vários grandes centros urbanos têm investido em programas de arborização de ruas, como Nova York - One Million trees (http://www.milliontreesnyc. org), já sendo estimada menor prevalência de asma infantil em $29 \%$ com o aumento da massa arbórea na cidade (Lovasi et al., 2008). Na cidade de São Paulo não existe um programa de arborização urbana que preveja adequadamente manejo e plantio. Sabemos ainda que existem muitos problemas na zeladoria e manutenção de áreas verdes da cidade, que certamente contribuem para o não uso da infraestrutura verde pela população. Esses problemas são ainda maiores nas áreas de maior vulnerabilidade social.

\section{Considerações finais}

Em estudos conduzidos principalmente na Europa e na América do Norte são muito concretas as evidências que associam presença/uso de áreas verdes com desfechos positivos de saúde. Proximidade de áreas verdes associa-se a menor obesidade, menor risco de desenvolver doença cardiovascular, menos doenças mentais, melhores desfechos de nascimento, entre outros. Além dos serviços ambientais prestados pelas áreas verdes, acredita-se que o contato com o verde diminua o estresse, aumente a coesão social e o nível de atividade física. Algumas grandes cidades do mundo têm investido em programas de arborização como maneira de melhorar a saúde urbana como um todo.

São Paulo tem áreas bastante desiguais de cobertura vegetal, sendo as maiores áreas situadas em regiões de maior vulnerabilidade social, localizadas nas bordas da cidade. Nas regiões mais centrais temos níveis muito baixos de cobertura vegetal, como nas subprefeituras da Sé e da Mooca, locais com altos níveis de poluição e ruído. A cidade sofre com a falta de planejamento na política de arborização urbana, seja no plantio, seja no manejo. As áreas verdes da cidade, como as praças, sofrem também com problemas de manutenção e zeladoria, e a fruição pública destas áreas pode ser prejudicada por esses motivos. Nas áreas mais desprovidas da cidade, há carência de ruas arborizadas e de equipamentos de lazer verde.

Estudos que investiguem a relação de áreas verdes e saúde em centros urbanos tão social e ambientalmente desiguais como a cidade de São Paulo são extremamente necessários, e podem contribuir na formação de políticas públicas que norteiem planos de arborização voltados a essa realidade.

\section{Notas}

l Embora não haja definições consensuais entre os termos, neste trabalho cobertura vegetal foi definida segundo a proposta da Prefeitura do Município de São Paulo (2014) como "áreas públicas e particulares com cobertura vegetal, incluindo áreas de vegetação arbórea, arbustiva e rasteira (gramíneas). Estão incluídas as áreas públicas com presença de vegetação, incluindo parques, praças, áreas vinculadas ao sistema viário (rotatórias, canteiros, etc.) e a equipamentos institucionais (cemitérios, escolas, universidades, entre outros), bem como as áreas vegetadas de propriedade particular (in- 
tralote), chácaras, sítios, reflorestamentos. Foram computadas também as áreas com ocorrência expressiva de mata nativa, em geral inseridas nas unidades de conservação de proteção integral (parques estaduais da Serra do Mar e da Cantareira)" e áreas verdes como "áreas de parques públicos urbanos municipais, parques estaduais urbanos, praças e todas as Unidades de Conservação de Proteção Integral definidas pelo Sistema Nacional de Unidades de Conservação - SNUC".

2 TMC são morbidades psíquicas de grande prevalência mundial, caracterizadas por sintomas como ansiedade, depressão, dificuldade de concentração e queixas somáticas, mas que não são enquadradas necessariamente dentro dos critérios do CID 10 (Classificação internacional de doenças) e do DMS-IV (Diagnostic and Statistical Manual of Mental Disorders).

3 Teste estatístico One-Way ANOVA para medidas repetidas, onde, $p=0.27$. Software IBM SPSS versão 22, IBM Corp., Chicago, IL, EUA.

4 IVP - "Aborda por setor censitário dimensões socioeconômicas como alfabetização e renda per capita, além de componentes demográficos como o ciclo de vida familiar (famílias jovens, adultas e idosas), localização (urbana ou rural) e aglomerados subnormais (favelas)" (Fundação Seade, 2013).

5 Parques públicos urbanos municipais, parques estaduais urbanos, praças e todas as Unidades de Conservação de Proteção Integral definidas pelo Sistema Nacional de Unidades de Conservação (SNUC), efetivamente implantadas na cidade.

\section{Agradecimentos}

Às agências financiadoras: Fapesp, CNPq e Capes, ao Instituto Nacional de Análise Integrada do Risco Ambiental (Inaira).

\section{Referências}

ABREU, R. N. Diagnóstico da cobertura arbórea da cidade de São Paulo. Relatório de Estágio Profissionalizante em Engenharia Florestal. Departamento de Ciências Florestais, Escola Superior de Agricultura "Luiz de Queiroz", 2013. 84p.

AFRANE, Y. et al. Effects of microclimatic changes caused by land use and land cover on duration of gonotrophic cycles of Anopheles gambiae (Diptera: Culicidae) in Western Kenya Highlands. Journal of Medical Entomology. v.42, p.974-80, 2005.

AGAY-SHAY, K. et al. Green spaces and adverse pregnancy outcomes. Occupational and Environmental Medicine, n.71, p.562, 2014.

ARAYA, R. et al. Common mental disorders and the built environment in Santiago, Chile. Brazilian Journal of Psychiatry, n.190, p.394-401, 2007.

ASTELL-BURT, T.; MITCHELL, R.; HARTIG, T. The association between green space and mental health varies across the life course: A longitudinal study. Journal of Epidemiology \& Community Health. v.68, n.6, p.578-83, 2014.

COUTTS, C.; HAHN, M. G. Infrastructure, Ecosystem Services, and Human Health. International Journal of Environmental Research and Public Health, n.12, v.8, 2015.

DADVAND, P. et al. Surrounding greenness and pregnancy outcomes in four Spanish birth cohorts. Environ Health Perspective, v.120, n.10, p.1481-7, 2012. 
DADVAND, P. et al. Risks and benefits of green spaces for children: a cross-sectional study of associations with sedentary behavior, obesity, asthma, and allergy. Environmental Health Perspectives, v.122, p.1329-35, 2014.

DADVAND, P. et al. Green spaces and cognitive development in primary schoolchildren. PNAS. v.112, n.26, p.7937-42, 2015.

DAVIES, Z. G. et al. Mapping an urban ecosystem service: quantifying above-ground carbon storage at a city-wide scale. Journal of Applied Ecology, v.48, n.5, p.1125-34, 2011.

DOBBERT, L. Y. Arborização na cidade de Campinas/SP - percepção e conforto. Arborização na cidade de Campinas/SP-percepção e conforto. 2015. 186p. Tese (Doutorado) - Universidade de São Paulo. São Paulo, 2015.

DONOVAN, G. H.; BUTRY, D. T. Trees in the city: Valuing street trees in Portland, Oregon. Landscape and Urban Planning, v.94, n.2, p.77-83, 2010.

ESCOBEDO, F. J.; ADAMS, D. C.; TIMILSINA, N. Urban forest structure effects on property value. Ecosystem Services, v.12, p.209-17, 2015.

FANG, C. F.; LING, D. L. Guidance for noise reduction provided by tree belts. Landscape and Urban Planning, v.71, n.1, p.29-34, 2005.

FUNDAÇÃO SEADE, Fundação Sistema Estadual de Análise de Dados. Secretaria de Planejamento e Desenvolvimento Regional. Índice Paulista de Vulnerabilidade Social. 2013. Disponível em: <http://indices-ilp.al.sp.gov.br/view/index. php?prodCod=2>. Acesso em: 1 fev. 2016.

GIDLÖF-GUNNARSSON, A., ÖHRSTRÖM, E. Noise and well-being in urban residential environments: the potential role of perceived availability to nearby green areas. Landscape and Urban Planning, v.83, p.115-26, 2007.

GRAZULEVICIENE, R. et al. The Influence of Proximity to City Parks on Blood Pressure in Early Pregnancy. International Journal of Environmental Research and Public Health, v.11, p.2958-72, 2014.

JAMES, P. et al. A review of the health benefits of greenness. Current Epidemiology Reports, v.2, p.131-42, 2015.

KWEON, B. S. et al. Green common spaces and the social integration of inner-city older adults. Environment and Behavior, v.30, n.6, p.832-58, 1998.

LOVASI, G. S. et al. Children living in areas with more street trees have lower prevalence of asthma. Journal of Epidemiology and Community health, v.62, n.7, p.647-9, 2008.

MAAS, J. et al. Green space, urbanity, and health: how strong is the relation? Journal of Epidemiology Community Health, v.60, p.587-92, 2006.

McPHERSON, E. G.; MUCHNICK, J. Effects of street tree shade on asphalt concrete pavement performance. 2005.

McPHERSON, E. G.; SCOTT, K. I.; SIMPSON, J. R. Estimating cost effectiveness of residential yard trees for improving air quality in Sacramento, California, using existing models. Atmospheric Environment, v.32, n.1, p.75-84, 1998.

McPHERSON, E. G.; SIMPSON, J. R. Potential energy savings in buildings by an urban tree planting programme in California. Urban Forestry \& Urban Greening, v.2, n.2, p.73-86, 2003. 
MORSE, R. A.; CALDERONE, N. W. The value of honey bees as pollinators of US crops in 2000. Bee Culture, v.128, p.1-15, 2000.

NAÇÕES UNIDAS. World Urbanization Prospects: The 2014 Revision. 1-32. 2004. Disponível em: <http://esa.un.org/unpd/wup/Highlights/WUP2014-Highlights. pdf>. Acesso em: 16 out. 2015.

NOWAK, D. J. et al. Tree and forest effects on air quality and human health in the United States. Environmental Pollution, v.193, p.119-29, 2014.

NOWAK, D. J.; CRANE, D. E.; STEVENS, J. C. Air pollution removal by urban trees and shrubs in the United States. Urban Forestry \& Urban Greening, v.4, n.3, p.115-23, 2006.

NOWAK, D. J.; DWYER, J. F. Understanding the benefits and costs of urban forest ecosystems: Urban and community forestry in the northeast. Springer Netherlands, 2007. p.25-46.

O'DELL, R. A.; TAHERI, M.; KABEL, R. L. A model for uptake of pollutants by vegetation. Journal of the Air Pollution Control Association, v.27, n.11, p.1104-9, 1977.

OLIVEIRA, S.; ANDRADE, H.; VAZ, T. The cooling effect of green spaces as a contribution to the mitigation of urban heat: A case study in Lisbon. Building and Environment, v.46, n.11, p.2186-94, 2011.

PAULEIT, S.; DUHME, F. Assessing the environmental performance of land cover types for urban planning. Landscape and Urban Planning, v.52, n.1, p.1-20, 2000.

PREFEITURA DA CIDADE DE SÃO PAULO. Dados e indicadores de meio ambiente. São Paulo, 2014. Disponível em: <http://www.prefeitura.sp.gov.br/cidade/secretarias/upload/planejamento/Verde10_2013.xls>. Acesso em: 3 fev. 2016.

PREFEITURA DO MUNICÍPIO DE SÃO PAULO. Dados demográficos dos distritos pertencentes as subprefeituras. Disponível em: <http://www.prefeitura.sp.gov. br/cidade/secretarias/subprefeituras/subprefeituras/dados_demograficos/index. php? $=12758>$. Acesso em: 25 fev. 2016.

ROLNIK, R. et al. O programa Minha Casa, Minha Vida nas regiões metropolitanas de São Paulo e Campinas: aspectos socioespaciais e segregação. Cadernos Metrópole, São Paulo, v.17, n.33, 2015.

SBAU - SOCIEDADE BRASILEIRA DE ARBORIZAÇÃO URBANA. Carta a Londrina e Ibiporã. Boletim Informativo, v.3, n.5, p.3, 1996.

SILVA FILHO, D. F. Classificação temática do Município de São Paulo a partir de ortofotos multiespectrais com ênfase na cobertura arbóreo arbustiva. s. d. Disponível em: <http://cmq.esalq.usp.br/wiki/doku.php?id=publico:novidades>.

SIMPSON, J. R. Urban forest impacts on regional cooling and heating energy use: Sacramento County case study. Journal of Arboriculture, v.24, p.201-14, 1998.

SOLECKI, W. D. et al. Mitigation of the heat island effect in urban New Jersey. Global Environmental Change Part B: Environmental Hazards, v.6, n.1, p.39-49, 2005.

TAKANO, T.; NAKAMURA, K.; WATANABE, M. Urban residential environments and senior citizens' longevity in megacity areas: the importance of walkable green spaces. Journal of Epidemiology \& Community Health, v.56, n.12, p.913-18, 2002. 
TAMOSIUNAS, A. et al. Accessibility and use of urban green spaces, and cardiovascular health: findings from a Kaunas cohort study. Environmental Health, v.13, n.1, p.1-11, 2014.

THOMPSON, C. W. et al. More green space is linked to less stress in deprived communities: Evidence from salivary cortisol patterns. Landscape and Urban Planning, v.105, n.3, 2012.

USDA Forest service. Urban Forest, 2016. Disponível em: <http://www.fs.fed.us/ managing-land/urban-forests>. Acesso em: 1 fev. 2016.

VAN DER BERG, M. et al. Visiting green space is associated with mental health and vitality: A cross-sectional study in four European cities. Health \& Place, v.38, p.8-15, 2016.

VAN DILLEN, S. M. et al. Greenspace in urban neighborhoods and residents' health: adding quality to quantity. Journal of Epidemiology \& Community Health, v.66, n.6, 2012.

VILLENEUVE, P. J. et al. A cohort study relating urban green space with mortality in Ontario, Canada. Environmental Research, v.115, p.51-8, 2012.

WILKER, E. H. et al. Green Space and Mortality Following Ischemic Stroke. Environmental Research, v.133, p.42-8, 2014.

YANG, F. et al. The investigation of noise attenuation by plants and corresponding noise-reducing spectrum. Journal of Environmental Health, v.8, p.8-15, 2010.

ZHANG, B. et al. Effect of urban green space changes on the role of rainwater runoff reduction in Beijing, China. Landscape and Urban Planning, v.140, p.8-16, 2015.

RESUMO - A falta de planejamento no processo de urbanização das grandes metrópoles tem produzido numerosas externalidades negativas, entre elas a supressão de sua cobertura vegetal e de áreas verdes. Inúmeros benefícios têm sido reportados sobre como a presença da vegetação no meio ambiente urbano favorece fatores ambientais, sociais e econômicos, influenciando na saúde da população. O presente trabalho busca apresentar uma série de estudos com enfoque na associação entre áreas verdes e saúde e também uma sucinta reflexão sobre a importância do assunto no município de São Paulo.

PALAVRAS-CHAVE: Urbanização, Saúde pública, Cobertura vegetal, Área verdes.

ABSTRACT - The lack of planning in the urbanization process of big cities worldwide has produced numerous negative externalities, including the suppression of vegetation and green spaces. Multiple benefits have been reported on how urban vegetation promotes and influences environmental, social and economic factors, as well as people's health. This paper presents a series of studies focusing on the association between urban vege- 
tation and human health, as well as a brief reflection on the importance of this issue in the city of São Paulo.

KEYWORDS: Urbanization, Public health, Urban vegetation, Green spaces.

Luis Fernando Amato-Lourenço é engenheiro ambiental, doutorando em Ciências pelo Departamento de Patologia da Faculdade de Medicina da Universidade de São Paulo, pesquisador-colaborador do Instituto Nacional de Análise Integrada do Risco Ambiental (Inaira) e do Grupo de Estudos em Agricultura Urbana (Geau).

@ - luisfamato@usp.br

Tiana Carla Lopes Moreiraé engenheira agrônoma e doutora em Ciências pelo Departamento de Patologia da Faculdade de Medicina da Universidade de São Paulo e pesquisadora-colaboradora do Instituto Nacional de Análise Integrada do Risco Ambiental (Inaira). @ - tianacarla@usp.br

Bruna Lara de Arantes é mestranda no programa de Recursos Florestais da Escola Superior de Agricultura "Luiz de Queiroz" (Esalq), da Universidade de São Paulo. @ - blarantes@usp.br

Demóstenes Ferreira da Silva Filho é professor associado da Escola Superior de Agricultura “Luiz de Queiroz” (Esalq), da Universidade de São Paulo. @ - dfilho@usp.br Thais Manad é professora associada da Faculdade de Medicina da Universidade de São Paulo, coordenadora do INCT Instituto Nacional de Análise Integrada do Risco Ambiental (Inaira). @-tmauad@usp.br

Recebido em 25.2.2016 e aceito em 15.3.2016.

I Faculdade de Medicina, Universidade de São Paulo, São Paulo/São Paulo, Brasil.

II Escola Superior de Agricultura “Luiz de Queiroz”, Universidade de São Paulo, Piracicaba/São Paulo, Brasil.

III Instituto Nacional de Análise Integrada do Risco Ambiental, São Paulo/São Paulo, Brasil. 